\title{
Influência da violência perpetrada por parceiro íntimo na prática do aleitamento
}

\section{materno}

\author{
Influence of violence perpetrated by an intimate partner in the practice of breastfeeding \\ Influencia de la violencia de pareja en la práctica de la lactancia materna
}

Recebido: 07/12/2021 | Revisado: 15/12/2021 | Aceito: 24/12/2021 | Publicado: 04/01/2022

Larissa Sales Martins Baquião
ORCID: https://orcid.org/0000-0002-7964-3935
Instituto Federal de Educação, Ciência e Tecnologia do Sul de Minas Gerais, Brasil
E-mail: larissa.martins@muz.ifsuldeminas.edu.br
Caroline Pagani Martins
ORCID: https://orcid.org/0000-0001-6217-4944
Universidade Estadual de Londrina, Brasil
E-mail:carol_pagani@hotmail.com
Wellington Pereira Lopes
ORCID: https://orcid.org/0000-0001-6997-9980
Universidade Estadual de Londrina, Brasil
E-mail:wellington-lopes@outlook.com
Lucas Lima de Moraes
ORCID: https://orcid.org/0000-0003-1613-5068
Universidade Estadual de Londrina, Brasil
E-mail:lucaslima16@outlook.com
Viviane Michele do Amaral
ORCID: https://orcid.org/0000-0002-0497-1272
Universidade Estadual de Londrina, Brasil
E-mail: viviane.amaral@uel.br
Sergio Valverde Marques dos Santos
ORCID: https://orcid.org/0000-0001-9412-9515
Universidade do Estado de Minas Gerais, Brasil
E-mail: sergio.valverde@uemg.br
Glilciane Morceli
ORCID: https://orcid.org/0000-0001-8216-9931
Universidade do Estado de Minas Gerais, Brasil
E-mail: glilciane.morceli@uemg.br

\section{Resumo}

Objetivo: Investigar as repercussões das diferentes formas de violência por parceiro íntimo (VPI) sobre as práticas de iniciação e continuidade do aleitamento materno (AM). Metodologia: Realizou-se uma revisão integrativa da literatura, entre 2008 a 2020 nas bases/bancos de dados PubMed, BVS e SciELO. Os descritores foram aleitamento materno, violência contra a mulher, amamentação e violência. A busca bibliográfica adotou a Metodologia Voltada para Pesquisa Clínica (PICO) e para selecionar e avaliar os artigos utilizou-se a estratégia Itens de Relatórios Preferidos para Analise Sistemática e Meta-Analise (PRISMA). Para verificar o nível de evidência dos estudos, utilizou-se o instrumento recomendado por Gershon e colaboradores (1999). Resultados e Discussão: Foram encontrados 40 artigos, destes 23 atenderam aos critérios de inclusão. Dos artigos selecionados, a maioria foi publicado entre 2018 e 2020. Conclusão: Evidenciou-se que associação entre o aleitamento materno e a violência por parceiro íntimo, acarreta em prejuízos ao início precoce, à manutenção e a exclusividade da amamentação.

Palavras-chave: Aleitamento materno; Violência por parceiro íntimo; Violência contra a mulher.

\section{Abstract}

The study aimed to investigate the repercussions of different forms of intimate partner violence on initiation and continuity practices of breastfeeding. An integrative literature review was carried out between 2008 and 2020 in the PubMed database, Virtual health Library (VHL) and Scientific Eletronic Library Online (SciELO). The descriptors used to search for the articles were breastfeeding, violence against women, breastfeeding and violence. To perform the bibliographic search, the Methodology Focused on Clinical Research was adopted and to select and evaluate the articles, the Preferred Reporting Items for Systematic reviews and Meta-Analyses was used as a strategy. To verify the level of evidence in the studies, the instrument recommended by Gershon et al. (1999) was used. Forty articles were found, of which 23 met the inclusion criteria. Of the selected articles, the majority were published between 2018 and 2020 . Of 
these, 11 had a cross-sectional design (48\%) and seven were cohort (30\%). It was evidenced that the association between breastfeeding and intimate partner violence, harms early initiation, maintenance and exclusivity of breastfeeding.

Keywords: Breastfeeding; Intimate partner violence; Violence against women.

\section{Resumen}

Objetivo: Investigar las repercusiones de las diferentes formas de violencia de pareja (VPI) en las prácticas de inicio y continuación de la lactancia materna. Metodología: Se realizó una revisión integradora de la literatura, entre 2008 y 2020 en las bases de datos PubMed, BVS y SciELO. Los descriptores fueron lactancia materna, violencia contra la mujer, lactancia materna y violencia. La búsqueda bibliográfica adoptó la Metodología Prospectiva para la Investigación Clínica (PICO) y se utilizó la estrategia de los Elementos de Información Preferidos para el Análisis Sistemático y el Metaanálisis (PRISMA) para seleccionar y evaluar los artículos. Para verificar el nivel de evidencia de los estudios, se utilizó el instrumento recomendado por Gershon y colaboradores (1999). Resultados y discusión: Se encontraron 40 artículos, 23 de los cuales cumplían los criterios de inclusión. De los artículos seleccionados, la mayoría fueron publicados entre 2018 y 2020. Conclusión: Se evidenció que la asociación entre la lactancia materna y la violencia de pareja perjudica el inicio temprano, el mantenimiento y la exclusividad de la lactancia materna.

Palabras clave: Lactancia Materna; Violencia de Pareja; Violencia contra la Mujer.

\section{Introdução}

O aleitamento materno (AM) é uma prática de destaque que contribui para o crescimento e o desenvolvimento saudável das crianças (Bandara et al., 2014; OMS, 2013). A Organização Mundial de Saúde (OMS), o Fundo das Nações Unidas para a Infância (Unicef) e o Ministério da Saúde (MS) recomendam que as crianças recebam exclusivamente o leite materno nos primeiros seis meses de vida (OMS, 2013). No entanto, pesquisas mostram que somente 37\% das crianças de todo o mundo são beneficiadas com este alimento dentro do período preconizado (Victora et al., 2016; Brasil, 2006).

Em muitos países, $80 \%$ dos recém-nascidos recebem AM. Contudo, ainda há uma alta prevalência de índices inferiores a 50\% relacionados ao aleitamento materno exclusivo (AME) até os seis meses de idade. Esse dado é preocupante, visto que essa prática, de forma adequada, pode prevenir a morte de mais de 800.000 mil crianças menores de 5 anos de idade por ano (Baraldi et al., 2020).

Práticas inadequadas de alimentação na infância podem acarretar obesidade ou desnutrição, sendo a última uma das principais causas de mortalidade na infância mundialmente. A prática da amamentação pode ter influência de vários fatores, que envolvem a idade materna, o nível de escolaridade, as condições socioeconômicas, os distúrbios mentais, a rede de apoio familiar e profissional e o ambiente em que a nutriz está inserida (Moraes et al., 2019).

O AME traz diversos benefícios para a lactante, como a redução de hemorragias, devido às contrações uterinas, espaçamento entre uma gestação e outra se o aleitamento for realizado em livre demanda e se a mulher se mantiver amenorreica e ainda a diminuição da probabilidade de desenvolver anemia, câncer de mama e ovário (Rea, 2004).

Pesquisas recentes demonstram associação entre a violência perpetrada por parceiro e as práticas inadequadas de AM (Chaves et al., 2019; Kjerulff Madsen et al., 2019; Normann et al., 2020). A violência praticada por parceiro íntimo se configura como condutas que provocam danos físicos, sexuais ou psicológicos à mulher, incluindo comportamento controlador. Apesar de ser a principal forma de violência que acomete a população feminina, ainda é bastante ocultada, sendo que somente uma pequena parcela das vítimas procura ajuda ou denuncia (McGuire, 2015).

A violência por parceiro íntimo (VPI) pode interferir de forma direta no aleitamento materno de várias maneiras, como dificuldade de relaxamento para descida em quantidade adequada do leite e dores na região do mamilo. Além disso, pode contribuir de forma indireta, por meio de falta de apoio emocional, depressão, psicose, ansiedade e até mesmo por descontentamento com o próprio corpo (Normann et al., 2020).

Diante do exposto, justifica-se a importância de investigar os estudos sobre as repercussões das diferentes formas de violências enfrentadas pelas mulheres durante a $\mathrm{AM}$, bem como promover informações sobre essa temática. Isto, devido ao fato desse tipo de situação causar prejuízos a mulher, ao bebê e ao processo de amamentação. Assim, torna-se relevante identificar 
na literatura científica os estudos relacionados a violência que a mulheres sofrem dos parceiros durante o período de amamentação.

Para isso, essa revisão integrativa teve como objetivo investigar as repercussões das diferentes formas de violência por parceiro íntimo (VPI) sobre as práticas de iniciação e continuidade do aleitamento materno.

\section{Metodologia}

Trata-se de uma revisão integrativa realizada a partir de um levantamento bibliográfico executado por meio da coleta de dados secundários. A revisão integrativa é uma ferramenta valiosa no campo da saúde, uma vez que sintetiza as pesquisas disponíveis acerca de determinada temática e orienta a prática baseada em conhecimento científico (Souza et al., 2010). É um dos métodos de pesquisa utilizados na Prática Baseada em Evidência (PBE), permitindo a incorporação das evidências na prática clínica (Mendes et al., 2008; Santos et al., 2007).

As etapas para o desenvolvimento desse estudo foram: a identificação do tema e seleção da questão de investigação de pesquisa; estabelecimento de critérios de inclusão e exclusão de artigos (busca da literatura); definição das informações a serem extraídas dos estudos; avaliação dos dados; análise e apresentação dos resultados; e apresentação da revisão (Mendes et al., 2008).

Para direcionar o estudo e alcançar os objetivos propostos, foi elaborada a seguinte pergunta: Quais as possíveis repercussões das VPI sobre as práticas do AM? Para responder essa pergunta, realizou-se as buscas dos artigos entre julho e novembro de 2020 nas seguintes bases de dados: USA National Library of Medicine (PubMed), Virtual Health Library (BVS) e Scientific Eletronic Library Online (Scielo). Os descritores utilizados para a busca dos artigos foram "aleitamento materno", "violência contra a mulher", "amamentação" e "violência" utilizando-se o operador booleano AND, nos seguintes cruzamentos: "aleitamento materno AND violência contra mulher" e "amamentação AND violência".

Para realizar a busca bibliográfica, foi adotada como estratégia a Metodologia Voltada para Pesquisa Clínica (PICO), representado da seguinte forma: $\mathrm{P}=$ Paciente/Problema, I=Intervenção ou indicadores, $\mathrm{C}=$ Comparação e $\mathrm{O}=$ Resultado/desfecho. Essa estratégia tem sido utilizada para construir questões de pesquisa de diferentes naturezas, provenientes da prática clínica (Santos et al., 2007).

Foram incluídos artigos disponíveis na íntegra, publicados em português, inglês ou espanhol, indexados nos referidos bancos de dados, publicados no período de 2008 a 2020, no mês de março de 2021 e relacionados à temática em estudo. Foram excluídos os trabalhos que não apresentavam associação entre as variadas formas de violência contra a mulher perpetradas por parceiro íntimo e a prática do aleitamento materno e estudos de revisão, bem como as duplicatas.

A seleção dos artigos foi realizada por meio do título e dos resumos e aqueles selecionados foram submetidos à leitura na íntegra e à análise minuciosa. Os dados extraídos das publicações selecionadas foram transcritos para um instrumento, construído pelos autores, para atender ao objetivo do estudo. O instrumento continha as seguintes variáveis: autores, título, desenho do estudo, amostra, periódico, país, ano de publicação e principais resultados.

Prosseguiu-se com a análise e a síntese das informações extraídas, de forma descritiva, permitindo observar, descrever e classificar os dados, com o propósito de sintetizar o conhecimento produzido acerca do tema explorado.

Com o objetivo de analisar os delineamentos metodológicos das pesquisas selecionadas e verificar suas qualidades, utilizou-se um instrumento para classificar os níveis de evidência científica extraídos das publicações, com base na proposta descrita por Gershon et al, 1999. Para a classificação do nível de evidência, consideraram-se os níveis 1 e 2 como evidências fortes, 3 e 4 moderadas e 5 a 7 fracas (Gershon et al., 1999; Goyatá et al., 2016). 
Com relação aos aspectos éticos, por se tratar de uma revisão integrativa da literatura e pelas informações secundárias serem extraídas de artigos científicos, já publicados em bases de dados e bibliotecas virtuais, não foi necessário a autorização para utilização dos dados e nem a apreciação do Comitê de Ética em Pesquisa, conforme a Resolução Nacional 466/2012.

\section{Resultados}

O percurso metodológico e a quantidade (n) de artigos científicos incluídos e excluídos em cada uma das etapas desse trabalho são apresentados na Figura 1.

Figura 1. Fluxograma de seleção de estudos sobre a relação entre a violência contra a mulher realizada por parceiro íntimo e o aleitamento materno para inclusão na revisão sistematizada da literatura. Brasil, 2021.

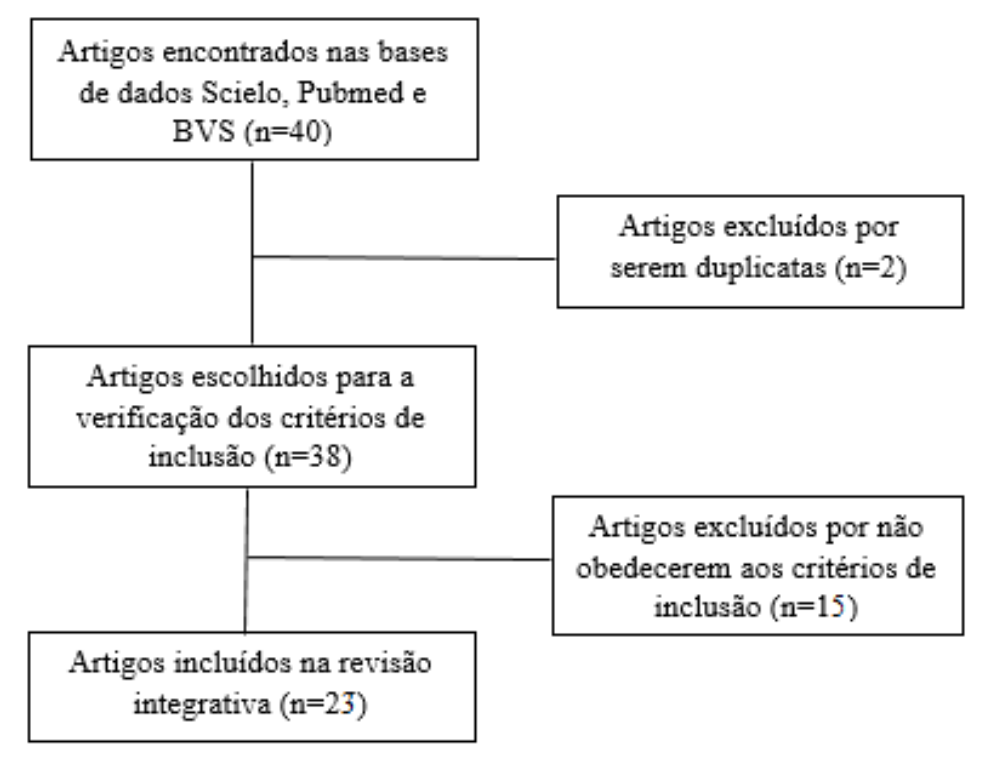

Fonte: Autores.

Os artigos incluídos nessa revisão integrativa a partir de informações relativas aos autores, título, desenho do estudo, amostra, periódico, país, ano de publicação e principais resultados são apresentados no Quadro 1. 
Quadro 1. Caracterização dos artigos incluídos na revisão quanto aos autores, título, desenho do estudo, amostra, periódico, país, ano de publicação e principais resultados. Brasil, 2021

\begin{tabular}{|c|c|c|c|c|c|c|c|}
\hline Autores & Título & $\begin{array}{l}\text { Desenho do } \\
\text { estudo }\end{array}$ & Amostra & Periódico & País & $\begin{array}{c}\text { Ano de } \\
\text { publicação }\end{array}$ & Principais resultados \\
\hline Mariano et al. & $\begin{array}{l}\text { Aleitamento materno exclusivo } \\
\text { e auto eficácia materna entre } \\
\text { mulheres em situação de } \\
\text { violência por parceiro íntimo }\end{array}$ & $\begin{array}{c}\text { Estudo } \\
\text { Transversal }\end{array}$ & 50 mulheres & $\begin{array}{c}\text { Texto \& } \\
\text { Contexto - } \\
\text { Enfermagem }\end{array}$ & Brasil & 2016 & $\begin{array}{l}\text { Não houve associação estatisticamente significativa entre a } \\
\text { duração do AME com } 30 \text { e } 70 \text { dias de pós-parto e a ocorrência } \\
\text { de tipos de VPI na gestação. Além disso, independentemente } \\
\text { do tipo de violência sofrida, o perfil de distribuição do nível } \\
\text { de auto eficácia entre as participantes não foi alterado. }\end{array}$ \\
\hline $\begin{array}{l}\text { Lourenço e } \\
\text { Deslandes }\end{array}$ & $\begin{array}{c}\text { Experiência do cuidado } \\
\text { materno e amamentação sob a } \\
\text { ótica de mulheres vítimas de } \\
\text { violência conjugal }\end{array}$ & $\begin{array}{c}\text { Pesquisa } \\
\text { Qualitativa } \\
\text { (Entrevista) }\end{array}$ & 11 mulheres & $\begin{array}{c}\text { Revista de } \\
\text { Saúde Pública }\end{array}$ & Brasil & 2008 & $\begin{array}{l}\text { O desmame ocorreu precocemente para a maioria das } \\
\text { entrevistadas. A necessidade de trabalhar fora, a falta de } \\
\text { informação sobre amamentação e a própria experiência de } \\
\text { violência foram as principais razões expostas para não } \\
\text { prosseguirem com a amamentação. }\end{array}$ \\
\hline Moraes et al. & $\begin{array}{l}\text { Prevalência de violência física } \\
\text { entre parceiros íntimos nos } \\
\text { primeiros seis meses após o } \\
\text { parto no Município do Rio de } \\
\text { Janeiro, Brasil }\end{array}$ & $\begin{array}{c}\text { Estudo } \\
\text { Transversal }\end{array}$ & $\begin{array}{l}927 \\
\text { mulheres/bebês }\end{array}$ & $\begin{array}{l}\text { Caderno de } \\
\text { Saúde Pública }\end{array}$ & Brasil & 2017 & $\begin{array}{l}\text { A VPI foi mais comum entre as mulheres que não estavam } \\
\text { oferecendo aleitamento materno exclusivo aos seus bebês. }\end{array}$ \\
\hline
\end{tabular}


Research, Society and Development, v. 11, n. 1, e18111124493, 2022

(CC BY 4.0) | ISSN 2525-3409 | DOI: http://dx.doi.org/10.33448/rsd-v11i1.24493

\begin{tabular}{|c|c|c|c|c|c|c|c|}
\hline Wallenborn et al. & $\begin{array}{l}\text { Association Between Intimate } \\
\text { Partner Violence and } \\
\text { Breastfeeding Duration: } \\
\text { Results From the 2004-2014 } \\
\text { Pregnancy Risk Assessment } \\
\text { Monitoring System }\end{array}$ & $\begin{array}{c}\text { Estudo } \\
\text { Transversal }\end{array}$ & $\begin{array}{l}195.264 \\
\text { mulheres }\end{array}$ & $\begin{array}{c}\text { Journal of } \\
\text { Human } \\
\text { Lactation }\end{array}$ & EUA & 2018 & $\begin{array}{l}\text { Mulheres que relataram terem sido vítimas de VPI antes da } \\
\text { gravidez apresentaram 1,29 vezes mais chances de nunca } \\
\text { amamentar e 1,27 vezes mais probabilidade de amamentar por } \\
8 \text { semanas ou menos em comparação com mulheres que não } \\
\text { relataram VPI. Sendo assim, houve associação entre VPI antes } \\
\text { da gravidez e práticas de amamentação abaixo do ideal entre } \\
\text { mulheres em idade reprodutiva. }\end{array}$ \\
\hline Boyce et al. & $\begin{array}{l}\text { Associations of intimate } \\
\text { partner violence with postnatal } \\
\text { health practices in Bihar, India }\end{array}$ & $\begin{array}{c}\text { Estudo } \\
\text { Transversal }\end{array}$ & 11.654 mulheres & $\begin{array}{c}\text { BMC } \\
\text { Pregnancy and } \\
\text { Childbirth }\end{array}$ & India & 2017 & $\begin{array}{l}\text { As chances de início precoce da amamentação diminuíram } \\
19 \% \text { e } 48 \% \text { entre as mulheres que experimentaram VPI física } \\
\text { e sexual, respectivamente. Já as chances de amamentação } \\
\text { exclusiva por } 6 \text { meses foram } 17 \% \text { menores entre as mulheres } \\
\text { que relataram VPI na forma física. }\end{array}$ \\
\hline Frith et al. & $\begin{array}{l}\text { Breast-feeding counselling } \\
\text { mitigates the negative } \\
\text { association of domestic } \\
\text { violence on exclusive breast- } \\
\text { feeding duration in rural } \\
\text { Bangladesh. The MINIMat } \\
\text { randomized trial }\end{array}$ & $\begin{array}{l}\text { Ensaio } \\
\text { Clínico }\end{array}$ & 3.186 mulheres & $\begin{array}{c}\text { Public Health } \\
\text { Nutrition }\end{array}$ & Bangladesh & 2017 & $\begin{array}{l}\text { O risco para a cessação precoce do AME foi maior entre as } \\
\text { mulheres do grupo que recebeu apenas mensagens de saúde } \\
\text { usuais e expostas a qualquer tipo de violência, sendo ainda } \\
\text { mais ampla entre as que vivenciaram um controle de } \\
\text { comportamento por parte do parceiro. No entanto, não houve } \\
\text { associação entre VPI e a duração do AM para as mulheres que } \\
\text { receberam mensagens com aconselhamentos específicos sobre } \\
\text { AM. }\end{array}$ \\
\hline Holland et al. & $\begin{array}{c}\text { Breastfeeding and Exposure to } \\
\text { Past, Current, and } \\
\text { Neighborhood Violence }\end{array}$ & $\begin{array}{c}\text { Estudo } \\
\text { Transversal }\end{array}$ & 760 mulheres & $\begin{array}{l}\text { Maternal and } \\
\text { Child Health } \\
\text { Journal }\end{array}$ & EUA & 2019 & $\begin{array}{l}\text { Para mulheres brancas, um histórico de exposição à violência } \\
\text { aumentou a probabilidade de interrupção precoce do AM, } \\
\text { enquanto que para as mulheres negras expostas à violência } \\
\text { houve um aumento da probabilidade de terem um plano de } \\
\text { amamentação e iniciá-la conforme o planejado. }\end{array}$ \\
\hline
\end{tabular}


Research, Society and Development, v. 11, n. 1, e18111124493, 2022

(CC BY 4.0) | ISSN 2525-3409 | DOI: http://dx.doi.org/10.33448/rsd-v11i1.24493

\begin{tabular}{|c|c|c|c|c|c|c|c|}
\hline $\begin{array}{l}\text { Martin-de-las- } \\
\text { Heras et al. }\end{array}$ & $\begin{array}{l}\text { Breastfeeding avoidance } \\
\text { following psychological } \\
\text { intimate partner violence } \\
\text { during pregnancy: a cohort } \\
\text { study and multivariate analysis }\end{array}$ & Coorte & 779 mulheres & BJOG & Espanha & 2018 & $\begin{array}{l}\text { Violência psicológica, relatada em } 1 \text { a cada } 5 \text { mulheres, esteve } \\
\text { associada com a redução no início da amamentação, sendo a } \\
\text { provável causa para tanto o estresse ao qual as mulheres são } \\
\text { submetidas nessas situações. Outros fatores que podem } \\
\text { influenciar no início tardio da amamentação são patologias e } \\
\text { complicações obstétricas. }\end{array}$ \\
\hline $\begin{array}{c}\text { Finnbogadóttir et } \\
\text { al. }\end{array}$ & $\begin{array}{l}\text { Breastfeeding in the context of } \\
\text { domestic violence - a cross } \\
\text { sectional study }\end{array}$ & $\begin{array}{c}\text { Estudo } \\
\text { Transversal }\end{array}$ & 731 mulheres & $\begin{array}{c}\text { Journal of } \\
\text { Advanced } \\
\text { Nursing }\end{array}$ & Suécia & 2017 & $\begin{array}{l}\text { Não houve diferença estatisticamente significativa entre os } \\
\text { grupos com ou sem história de VPI em relação ao AME. } \\
\text { Contudo, os resultados mostraram que mulheres com histórico } \\
\text { de violência apresentaram significativamente mais sintomas } \\
\text { de depressão do que aquelas sem, sendo que as que tinham } \\
\text { sintomas depressivos amamentaram de forma significativa e } \\
\text { exclusiva em menor grau aos dois e aos quatro meses. }\end{array}$ \\
\hline Caprara et al. & $\begin{array}{l}\text { Does domestic violence during } \\
\text { pregnancy influence the } \\
\text { beginning of complementary } \\
\text { feeding? }\end{array}$ & Coorte & 232 mulheres & $\begin{array}{c}\text { BMC } \\
\text { Pregnancy and } \\
\text { Childbirth }\end{array}$ & Brasil & 2020 & $\begin{array}{l}\text { Não foi encontrada associação significativa entre alimentação } \\
\text { complementar precoce e VPI durante a gravidez, sendo que } \\
\text { maioria das participantes que iniciaram a alimentação } \\
\text { complementar precocemente não relatou violência durante a } \\
\text { gestação. }\end{array}$ \\
\hline James et al. & $\begin{array}{l}\text { Does intimate partner violence } \\
\text { impact on women's initiation } \\
\text { and duration of breastfeeding? }\end{array}$ & $\begin{array}{l}\text { Ensaio clínico } \\
\text { randomizado } \\
\text { pragmático }\end{array}$ & 2.631 mulheres & $\begin{array}{c}\text { Breastfeeding } \\
\text { Review }\end{array}$ & Austrália & 2014 & $\begin{array}{l}\text { Não foram encontradas diferenças estatisticamente } \\
\text { significativas entre as mulheres que relataram VPI e aquelas } \\
\text { que não relataram em relação à amamentação. Contudo, a } \\
\text { amostra era composta predominantemente por mulheres com } \\
\text { idade materna avançada, com alto nível de escolaridade, } \\
\text { predominantemente casadas e com renda mais alta. A } \\
\text { prevalência de VPI, portanto, pode estar subestimada. }\end{array}$ \\
\hline
\end{tabular}


Research, Society and Development, v. 11, n. 1, e18111124493, 2022

(CC BY 4.0) | ISSN 2525-3409 | DOI: http://dx.doi.org/10.33448/rsd-v11i1.24493

\begin{tabular}{|c|c|c|c|c|c|c|c|}
\hline Islam et al. & $\begin{array}{l}\text { Exploring the influence of } \\
\text { psychosocial factors on } \\
\text { exclusive breastfeeding in } \\
\text { Bangladesh }\end{array}$ & $\begin{array}{c}\text { Estudo } \\
\text { Transversal }\end{array}$ & 426 mulheres & $\begin{array}{l}\text { Archives of } \\
\text { Women's } \\
\text { Mental Health }\end{array}$ & Bangladesh & 2016 & $\begin{array}{l}\text { Mulheres vítimas de VPI na forma física durante os primeiros } \\
6 \text { meses após o parto apresentaram um risco } 83,0 \% \text { maior de } \\
\text { interromper o AME. Já as que sofreram VPI na forma } \\
\text { psicológica após o parto obtiveram } 49 \% \text { menos probabilidade } \\
\text { de amamentar exclusivamente. }\end{array}$ \\
\hline Lau e Chan & $\begin{array}{l}\text { Influence of Intimate Partner } \\
\text { Violence During Pregnancy } \\
\text { and Early Postpartum } \\
\text { Depressive Symptoms on } \\
\text { Breastfeeding Among Chinese } \\
\text { Women in Hong Kong }\end{array}$ & $\begin{array}{c}\text { Estudo } \\
\text { Transversal }\end{array}$ & $\begin{array}{c}1.200 \\
\text { mulheres/bebês }\end{array}$ & $\begin{array}{c}\text { Journal of } \\
\text { Midwifery \& } \\
\text { Woman's } \\
\text { Health }\end{array}$ & China & 2010 & $\begin{array}{l}\text { Mulheres que sofreram agressão psicológica e coerção física } \\
\text { foram mais encontradas no grupo de alimentação artificial do } \\
\text { que nos grupos de amamentação e alimentação mista. Além } \\
\text { disso, mulheres que não sofreram VPI durante a gravidez } \\
\text { tinham maior probabilidade de iniciar a amamentação em } \\
\text { comparação com as que relataram abuso. }\end{array}$ \\
\hline Miller-Graff et al. & $\begin{array}{l}\text { Intimate Partner Violence and } \\
\text { Breastfeeding Outcomes in a } \\
\text { Sample of Low-Income } \\
\text { Women }\end{array}$ & Coorte & 101 mulheres & $\begin{array}{c}\text { Journal of } \\
\text { Human } \\
\text { Lactation }\end{array}$ & EUA & 2018 & $\begin{array}{l}\text { A VPI diminuiu a probabilidade de que as mulheres } \\
\text { estivessem amamentando } 6 \text { semanas após o parto, apoiando a } \\
\text { hipótese inicial de que a VPI interrompe precocemente a } \\
\text { amamentação. Contudo, quando se analisou a iniciação e a } \\
\text { exclusividade da amamentação não foi encontrada diferença } \\
\text { estatisticamente significativa entre as mulheres que sofreram } \\
\text { VPI e as que não sofreram. }\end{array}$ \\
\hline Normann et al. & $\begin{array}{c}\text { Intimate partner violence and } \\
\text { breastfeeding: a systematic } \\
\text { review }\end{array}$ & $\begin{array}{c}\text { Revisão } \\
\text { Sistemática }\end{array}$ & $\begin{array}{c}16 \text { estudos ( } 11 \\
\text { transversais e } 5 \\
\text { coorte) }\end{array}$ & BMJ Open & Dinamarca & 2020 & $\begin{array}{l}\text { Na maioria dos estudos foi encontrada associação entre o } \\
\text { início tardio ou a interrupção precoce da amamentação } \\
\text { exclusiva e a VPI. Além disso, o estudo traz a discussão que } \\
\text { mulheres que não sofreram VPI, mas que sofreram violência } \\
\text { na infância também podem apresentar dificuldades para } \\
\text { amamentar. }\end{array}$ \\
\hline
\end{tabular}




\begin{tabular}{|c|c|c|c|c|c|c|c|}
\hline Madsen et al. & $\begin{array}{l}\text { Intimate partner violence and } \\
\text { subsequent premature } \\
\text { termination of exclusive } \\
\text { breastfeeding: A cohort study }\end{array}$ & Coorte & 1.129 mulheres & PLOS One & Tanzania & 2019 & $\begin{array}{l}\text { Mulheres que foram expostas à VPI tiveram chance mais de } \\
\text { 50\% maior de encerrar a AME antes de a criança completar } 6 \\
\text { meses de idade em comparação com mulheres que não foram } \\
\text { expostas. Além disso, as chances eram triplicadas se a } \\
\text { exposição acontecesse especificamente durante a gravidez. }\end{array}$ \\
\hline Chaves et al. & $\begin{array}{l}\text { Intimate partner violence } \\
\text { identified through routine } \\
\text { antenatal screening and } \\
\text { maternal and perinatal health } \\
\text { outcomes }\end{array}$ & Coorte & 52.509 mulheres & $\begin{array}{c}\text { BMC } \\
\text { Pregnancy and } \\
\text { Childbirth }\end{array}$ & Austrália & 2019 & $\begin{array}{l}\text { VPI foi associada a um risco aumentado de depressão pós- } \\
\text { parto, não amamentação ao nascer, aleitamento materno não } \\
\text { exclusivo na alta hospitalar e na primeira visita após o } \\
\text { nascimento do bebê. Já o medo auto relatado de um parceiro } \\
\text { foi fortemente associado a um risco aumentado de ausência de } \\
\text { início precoce da amamentação e amamentação não exclusiva } \\
\text { na alta hospitalar e na primeira visita após o parto. }\end{array}$ \\
\hline Tran et al. & $\begin{array}{l}\text { Intimate partner violence is } \\
\text { associated with poorer } \\
\text { maternal mental health and } \\
\text { breastfeeding practices in } \\
\quad \text { Bangladesh }\end{array}$ & $\begin{array}{c}\text { Estudo } \\
\text { Transversal }\end{array}$ & 2.000 mulheres & $\begin{array}{l}\text { Health Policy } \\
\text { and Planning }\end{array}$ & Bangladesh & 2020 & $\begin{array}{l}\text { Mulheres expostas a VPI tiveram 2-2,3 vezes mais chances de } \\
\text { sofrerem de altos níveis de algum transtorno mental e são } 28- \\
34 \% \text { menos propensas a amamentar exclusivamente seus } \\
\text { bebês. }\end{array}$ \\
\hline Caleyachetty et al. & $\begin{array}{l}\text { Maternal exposure to intimate } \\
\text { partner violence and } \\
\text { breastfeeding practices in } 51 \\
\text { low-income and middle- } \\
\text { income countries: A } \\
\text { population-based cross- } \\
\text { sectional study }\end{array}$ & $\begin{array}{c}\text { Estudo } \\
\text { Transversal } \\
\text { de base } \\
\text { populacional }\end{array}$ & $\begin{array}{l}\text { Variou entre } \\
95.320 \text { e } \\
102.318 \\
\text { mulheres de } 51 \\
\text { países }\end{array}$ & $\begin{array}{c}\text { PLOS } \\
\text { Medicine }\end{array}$ & Reino Unido & 2019 & $\begin{array}{l}\text { Mães expostas a qualquer forma de VPI (física, sexual ou } \\
\text { emocional) tiveram menor probabilidade de iniciar a } \\
\text { amamentação precocemente e amamentar exclusivamente nos } \\
\text { primeiros } 6 \text { meses. }\end{array}$ \\
\hline
\end{tabular}




\begin{tabular}{|c|c|c|c|c|c|c|c|}
\hline Sørbø et al. & $\begin{array}{l}\text { Past and recent abuse is } \\
\text { associated with early cessation } \\
\text { of breast feeding: results from } \\
\text { a large prospective cohort in } \\
\text { Norway }\end{array}$ & Coorte & 53.934 mulheres & BMJ Open & Noruega & 2015 & $\begin{array}{l}\text { A exposição a abusos anteriores e recentes foi fortemente } \\
\text { associada à interrupção precoce de qualquer amamentação. O } \\
\text { efeito mais forte foi observado em mulheres expostas a três } \\
\text { tipos de abuso (sexual, físico e emocional), com aumento de } \\
\text { quase } 50 \% \text { nas razões de chances (odds ratio) ajustadas de } \\
\text { qualquer interrupção da amamentação antes de } 4 \text { meses em } \\
\text { comparação com as mulheres não expostas. }\end{array}$ \\
\hline Ribeiro et al. & $\begin{array}{c}\text { Recurrent Violence, Violence } \\
\text { with Complications, and } \\
\text { Intimate Partner Violence } \\
\text { Against Pregnant Women and } \\
\text { Breastfeeding Duration }\end{array}$ & Coorte & 1.146 mulheres & $\begin{array}{c}\text { Journal of } \\
\text { Women's } \\
\text { Health }\end{array}$ & Brasil & 2020 & $\begin{array}{l}\text { O risco de um bebê não ser amamentado nos primeiros } 12 \\
\text { meses de vida aumentou em casos de violência física, sexual e } \\
\text { psícológica recorrentes ocorridos anteriormente ou durante a } \\
\text { gravidez. }\end{array}$ \\
\hline Moraes et al. & $\begin{array}{l}\text { Severe physical violence } \\
\text { between intimate partners } \\
\text { during pregnancy: a risk factor } \\
\text { for early cessation of exclusive } \\
\text { breastfeeding }\end{array}$ & $\begin{array}{c}\text { Estudo } \\
\text { Transversal }\end{array}$ & 811 mulheres & $\begin{array}{c}\text { Public Health } \\
\text { Nutrition }\end{array}$ & Brasil & 2011 & $\begin{array}{l}\text { A probabilidade de crianças permanecerem em AME entre as } \\
\text { mulheres não expostas a episódios de violência severa durante } \\
\text { a gestação é consistentemente maior do que entre as mulheres } \\
\text { expostas durante todo o período pós-natal. }\end{array}$ \\
\hline Baraldi et al. & $\begin{array}{l}\text { The meaning of the social } \\
\text { support network for women in } \\
\text { situations of violence and } \\
\text { breastfeeding }\end{array}$ & $\begin{array}{c}\text { Pesquisa } \\
\text { Qualitativa } \\
\text { (Entrevista) }\end{array}$ & 21 mulheres & $\begin{array}{l}\text { Revista Latino } \\
\text { Americana de } \\
\text { Enfermagem }\end{array}$ & Brasil & 2020 & $\begin{array}{l}\text { Das } 21 \text { participantes, nove afirmaram ter sofrido algum tipo de } \\
\text { VPI durante a gravidez (violência psicológica, sexual e física e } \\
\text { psicológica associadas). No período pós-parto, } 5 \text { sofreram um } \\
\text { único tipo de violência, enquanto as demais foram vítimas de } \\
\text { dois ou mais tipos. Em relação ao AM, das } 21 \text { participantes, } \\
\text { apenas uma permaneceu em AME até o sexto mês. Ao final de } \\
180 \text { dias pós-parto, sete bebês estavam já desmamados, } \\
\text { enquanto } 13 \text { estavam com amamentação complementar. }\end{array}$ \\
\hline
\end{tabular}

Fonte: Autores. 
Em relação ao desenho dos estudos, dos 23 artigos incluídos nessa revisão, um consistia em uma revisão sistemática, 11 eram estudos transversais, sete eram de coorte, dois eram ensaios clínicos e dois configuravam pesquisas qualitativas realizadas por meio de entrevistas.

No que se refere aos locais de pesquisa, o Brasil foi o país mais prevalente, aparecendo em sete estudos. Em seguida vieram Estados Unidos $(n=3)$, Bangladesh $(n=3)$, Austrália $(n=2)$ e, com um estudo em cada, China, Suécia, Noruega, Dinamarca, Tanzânia, Espanha, Índia e Reino Unido. A VPI foi associada com a redução da intenção de amamentar em um estudo, quadros de desmame precoce em 20 estudos e menor probabilidade de iniciar o aleitamento materno em oito deles. Cinco estudos não encontraram associação significativa entre o início e a duração da amamentação e a VPI.

Ao analisar o nível de evidência dos estudos selecionados por meio da proposta de Gershon et al., 1999 conforme apresentado no Quadro 2, constatou-se que 13 (56,52\%) dos estudos apresentaram nível de evidência 6, considerado baixo, uma vez que suas abordagens eram descritivas ou qualitativas. Entre os demais estudos, um $(4,34 \%)$ consistia em um ensaio clínico randomizado, considerado como nível 2 de evidência, um (4,34\%) era um ensaio clínico, com nível 3 de evidência, sete (30,43\%) eram estudos de coorte, cujo nível de evidência é 4 e um $(4,34 \%)$ era uma revisão sistemática, com nível 5 de evidência.

Quadro 2- Distribuição dos estudos em relação à classificação do nível de evidência das publicações. (n = 23), Brasil, 2020.

\begin{tabular}{|c|c|c|c|}
\hline Tipo de estudo & $\begin{array}{c}\text { Nível de } \\
\text { evidência** }\end{array}$ & $\mathbf{N}$ & $\%$ \\
\hline $\begin{array}{l}\text { Meta-análise, Revisão Sistemática de Ensaio Clínico } \\
\text { Randomizado, Ensaio Clínico Randomizado Controlado }\end{array}$ & 1 & - & - \\
\hline $\begin{array}{l}\text { Pelo menos um Ensaio Clínico Randomizado Controlado bem } \\
\text { delineado }\end{array}$ & 2 & 1 & 4,34 \\
\hline Ensaio Clínico, sem randomização & 3 & 1 & 4,34 \\
\hline Estudo de Coorte, Estudo Caso-controle & 4 & 7 & 30,43 \\
\hline Revisão Sistemática de estudos descritivos ou qualitativos & 5 & 1 & 4,34 \\
\hline Estudo descritivo ou qualitativo & 6 & 13 & 56,52 \\
\hline Opinião de autoridades ou Relatório de Comitê de Especialistas & 7 & - & - \\
\hline Total & & 23 & $100 \%$ \\
\hline
\end{tabular}

Fonte: Gershon, et al., (1999) ; Goyatá et al., (2016).

\section{Discussão}

Entre os Objetivos de Desenvolvimento Sustentável preconizados pela Organização das Nações Unidas em 2015, se destaca como uma das prioridades, no âmbito da saúde e bem-estar, a mitigação das mortes evitáveis de recém-nascidos em todo o mundo, de modo que a mortalidade neonatal seja reduzida para no máximo 12 por 1.000 nascidos vivos até o 
ano de 2030 (ONU, 2015). Para tanto, é fundamental que práticas reconhecidamente viáveis e efetivas na promoção da saúde neonatal, como o início precoce da amamentação, sejam amplamente difundidas. Nesse sentido, Victora et al., 2016 fomentam que o aleitamento materno está diretamente relacionado à redução na mortalidade súbita de bebês, bem como na proteção contra doenças crônicas, como a diabetes.

Há uma vasta gama de benefícios do AM tanto para as mães quanto para as crianças, inclusive nos lares onde a VPI está presente, conforme demonstram Miller-Graff et al., 2018 ao identificarem que a exposição à VPI não exerceu efeito negativo sobre o temperamento das crianças aos 4 meses nos casos em que as mães amamentavam por pelo menos seis semanas após o parto. Sendo assim, recomenda-se que o AME seja realizado durante os primeiros seis meses de vida do bebê e, mesmo após esse período, quando a alimentação complementar for introduzida, a amamentação deve ser continuada por um ano ou mais (Krug et al., 2002).

A discussão sobre a problemática da VPI ocorrida antes, durante e/ou depois da gestação, perpassa, primeiramente, as diferentes formas como esta pode se manifestar. A tipologia da violência proposta no Relatório Mundial sobre Violência e Saúde da OMS caracteriza a violência física como atitudes violentas com o uso da força física de modo intencional, com intuito de ferir, lesar, provocar dor e sofrimento ou até mesmo destruir a pessoa. A violência sexual é qualquer ação na qual uma pessoa obriga a outra a ter, presenciar, ou participar de interações sexuais ou a utilizar a sua sexualidade com fins lucrativos. Violência psicológica é qualquer forma de rejeição, depreciação, discriminação, desrespeito, cobrança exagerada, controle de ações e punições humilhantes usando a intimidação, manipulação ou ameaça e a negligência é definida como a omissão em prover as necessidades e cuidados básicos para o desenvolvimento físico, emocional e social da pessoa. De acordo com a Organização Mundial de Saúde (OMS., 2013), um terço das mulheres em todo o mundo já vivenciaram uma ou mais formas de VPI em suas vidas.

Em estudo de coorte realizado com 53.934 mulheres na Noruega, identificou-se que a exposição à violência física predispõe ao risco de interrupção precoce da amamentação independentemente de haver depressão pré ou pós-parto e outros fatores intermediários (Sorbo et al., 2015). Já na pesquisa de Boyce et al., 2017 a ocorrência de abuso sexual foi amplamente relatada entre mães que não iniciaram o AM nos primeiros dias de vida do recém nascido, enquanto que as agressões físicas estavam associadas a menor prevalência de AME. Resultados semelhantes foram encontrados por Tesfahun et al., $2013^{24}$ os quais avaliaram que a experiência de VPI nas formas física ou sexual, em algum momento da vida, foi associada a um menor quantitativo de mulheres amamentando exclusivamente seus bebês até os 6 meses de idade.

Contudo, embora muitos estudos de VPI tenham como foco as formas físicas e sexuais de abuso, a VPI no aspecto psicológico não pode ser ignorada, tendo em vista que estudos demonstram que essa modalidade de violência pode ser tão prejudicial quanto as demais. No estudo de Islam et al., 2016, por exemplo, o abuso psicológico foi o tipo mais comum de VPI vivido após o parto, segundo o relato das parturientes, sendo que as vítimas desse tipo de VPI apresentaram 49\% menos probabilidade de amamentarem exclusivamente seus filhos. Em outra pesquisa, realizada na Espanha, foi observado que a violência psicológica, relatada em 1 a cada 5 mulheres, estava associada com a redução das chances de se iniciar a amamentação (Martin-de-las-Heras et al., 2019).

Há dois mecanismos que explicam o modo como a VPI impacta o período perinatal. O primeiro, referente a uma associação direta, reflete o trauma em si, promovendo lesões na mulher que repercutem na gestação, enquanto que o segundo é indireto e baseado na teoria do estresse contínuo. Isto é, o estresse provocado pela vivência prolongada de violência reduz a capacidade da mulher em cuidar de si, repousando pouco e mantendo uma alimentação inadequada, além de frequentar menos os serviços de saúde. Tendo em vista que os níveis de cortisol da parturiente são diminuidos em virtude das agressões, o 
suprimento de leite após o parto é retardado e, por pensar que está produzindo uma quantidade inadequada do líquido, a mãe interrompe o AME (Kendall-Tackett, 2007).

Além de lesões físicas, a violência contra a mulher pode gerar incapacitação, depressão, interferências na saúde reprodutiva, gravidez indesejada, aborto inseguro, impulsionar o uso de álcool, tabaco e drogas, gerar comportamento sexual de alto risco, câncer e doenças crônicas e infecciosas, bem como morte prematura (OMS, 2014).

Silverman et al., 2006 afirmam que a presença de um histórico de VPI ampliam as chances de episódios de ansiedade e depressão, os quais impactam negativamente na saúde sexual, reprodutiva e no pós-parto, havendo em muitos casos interrupção precoce da amamentação. Ressalta-se ainda que mulheres que estavam deprimidas durante a gravidez costumeiramente se mostram deprimidas também durante o período pós-parto.

Kendall-Tackett et al., 2007 ainda destacam que algumas barreiras relacionadas à amamentação são mais prevalentes em relacionamentos abusivos, como a falta de apoio do parceiro, menor permanência na maternidade após o parto, partos prematuros e baixo peso ao nascer. Os abusadores, por sua vez, comumente promovem entraves para a ida das gestantes às consultas de pré-natal, pois temem que as práticas violentas sejam reveladas. Assim, informações e orientações importantes sobre as vantagens da amamentação e prevenção acerca da violência contra mulher são repassadas tardiamente ou nem ao menos chegam ao conhecimento das mulheres, reduzindo-se assim a probabilidade de que o AM seja sequer iniciado ou a violência seja prevenida (McFarlane et al., 1995; Ferrari et al., 2020).

Mesmo nos estudos onde não foi encontrada associação entre VPI e prejuízos à amamentação, faz-se necessário discutir alguns aspectos. Finnbogadóttir et al., 2017, por exemplo, não encontraram diferença estatisticamente significativa entre os grupos com ou sem história de violência em relação ao AM. No entanto, mulheres com histórico de violência apresentaram significativamente mais sintomas de depressão do que aquelas sem, sendo que elas amamentaram de forma significativa e exclusiva em menor grau do que as que possuíam menos sintomas da doença.

Ainda nesse sentido, James et al., 2020 alertam para o aspecto socio-econômico do problema ao discutirem que as mulheres entrevistadas em seu estudo em geral apresentavam idade materna avançada, alto nível de escolaridade, renda mais alta e eram predominantemente casadas (Campos et al., 2021). A prevalência de VPI, portanto, poderia estar subestimada, já que a violência doméstica durante a gravidez é significativamente mais prevalente em mulheres com baixa escolaridade (James et al., 2020). Além disso, as mulheres em situação de vulnerabilidade socioeconômica são mais propensas a precisar de recursos adicionais para se proteger e a seus filhos da violência (Silverman et al., 2006).

É preciso compreender a violência em suas variadas formas de apresentação e desenvolver ações em busca de seu enfrentamento. A VPI deve ser incluída na agenda das políticas e programas de saúde ligados ao aleitamento materno, focando não somente no recém-nascido, mas também na mãe (Bueno \& Teruya, 2004). Entretanto, em estudo recente foi observado que embora alguns países como Espanha, Brasil, Índia, Líbano e África do Sul tenham desenvolvido protocolos e diretrizes que incluem a VPI como parte dos cuidados durante o pré-natal, a sua implantação nos respectivos sistemas tem progredido de forma lenta (Palhoni et al., 2014; Tran et al., 2020).

Cabe aos serviços e aos profissionais de saúde, especialmente aos enfermeiros, a tarefa de acompanhar, incentivar e orientar as gestantes sobre os benefícios da amamentação. Neste processo de cuidado, no entanto, é fundamental que estejam atentos para não causarem ainda mais sofrimento às mulheres, revitimizando-as. Para tanto, os profissionais devem ser capacitados para a abordagem do tema de maneira adequada, auxiliando na promoção da autoestima e da autoconfiança dessas mulheres (Bueno \& Teruya, 2004).

No que se refere à prática baseada em evidências, destaca-se o uso de diferentes sistemas para classificar a qualidade dos achados em cada estudo, isto é, seu nível de evidência. De maneira usualmente hierárquica, as pesquisas são caracterizadas 
a partir da abordagem metodológica adotada (Galvão, 2006). Nesse sentido, é fundamental salientar que esse processo de classificação pode variar dependendo tanto do instrumento utilizado quanto do avaliador e, além disso, o fato de alguns estudos terem níveis de evidência considerados mais baixos não necessariamente exclui o seu potencial de prover recomendações importantes (Guyatt, Oxman, Kunz, Falck-Ytter, et al., 2008; Guyatt, Oxman, Kunz, Vist, et al., 2008).

Por fim, sugere-se que trabalhos futuros aprofundem os conhecimentos sobre a temática das repercussões da VPI no AM, valendo-se de revisões sistemáticas de literatura.

\section{Conclusão}

Esta revisão revela que a associação entre o AM e a VPI, encontrada na maioria dos estudos, observando-se prejuízos ao inicío precoce, à manutenção e a exclusividade da amamentação. Os estudos que não encontraram associação estatística entre AM e VPI demonstraram maior probabilidade dessas mulheres desenvolverem sintomas de depressão após o parto, sendo que muitas delas acabaram amamentando em menor grau do que as que não sofriam nenhum tipo de violência.

Outro ponto a ser destacado é a falta de protocolos e ações estratégicas de enfrentamento a essa realidade durante o pré-natal e no pós-parto, visto que a maioria dos países não tem nenhum planejamento que envolva educação continuada ou permanente dos trabalhadores da área da saúde nesse sentido. Além disso, os países que têm protocolos registrados reveleram uma progresssão lenta nas intervenções de proteção a mulher e na potencialização do AME em meio a VIP, sendo necessário uma atenção maior à essa temática.

Por fim, evidenciou-se que a maioria das produções científicas que relacionam AM e VIP aprensentam nível de evidência fraco. Por esse motivo, mostra-se imprescindível a realização de novas pesquisas com abordagens que apresentem maiores níveis de evidência, assim como se espera que esse artigo possa contruibuir para um aprofundamento e melhor compreensão do tema estudado.

\section{Referências}

Bandara, T., Hettiarachchi, M., Liyanage, C., \& Amarasena, S. (2014). Current infant feeding practices and impact on growth in babies during the second half of infancy. Journal of Human Nutrition and Dietetics, 28(4), 366-374. https://doi.org/10.1111/jhn.12253

Baraldi, N. G., Lettiere-Viana, A., Carlos, D. M., Salim, N. R., Pimentel, D. T. R., \& Stefanello, J. (2020). The meaning of the social support network for women in situations of violence and breastfeeding. Revista Latino-Americana de Enfermagem, 28. https://doi.org/10.1590/1518-8345.3313.3316

Boyce, S. C., McDougal, L., Silverman, J. G., Atmavilas, Y., Dhar, D., Hay, K., \& Raj, A. (2017). Associations of intimate partner violence with postnatal health practices in Bihar, India. BMC Pregnancy and Childbirth, 17(1). https://doi.org/10.1186/s12884-017-1577-0

Breastfeeding and the use of human milk. (2012). PEDIATRICS, 129(3), e827-e841. https://doi.org/10.1542/peds.2011-3552

Bueno, L. G. dos S., \& Teruya, K. M. (2004). The practice of breastfeeding counseling. Jornal de Pediatria, 80(8), 126-130. https://doi.org/10.2223/1243

Campos, L. A. de, Polettini, J., Moraes, L. L. de, Silva, B. D. M. da, Saek, E. K., Ferrari, D. V. de J., \& Morceli, G. (2021). Via de parto: Influência no teor de gorduras do colostro de nutrizes em maternidade do interior do Estado de São Paulo. Research, Society and Development, 10(2), e10210212165. https://doi.org/10.33448/rsd-v10i2.12165

Caprara, G. L., Bernardi, J. R., Bosa, V. L., da Silva, C. H., \& Goldani, M. Z. (2020). Does domestic violence during pregnancy influence the beginning of complementary feeding? BMC Pregnancy and Childbirth, 20(1). https://doi.org/10.1186/s12884-020-03144-y

Chaves, K., Eastwood, J., Ogbo, F. A., Hendry, A., Jalaludin, B., Khanlari, S., \& Page, A. (2019). Intimate partner violence identified through routine antenatal screening and maternal and perinatal health outcomes. BMC Pregnancy and Childbirth, 19(1). https://doi.org/10.1186/s12884-019-2527-9

Ferrari, D. V. de J., Polettini, J., de Moraes, L. L., de Campos, L. A., da Silva, M. G., Saeki, E. K., \& Morceli, G. (2020). Profile of pro-inflammatory cytokines in colostrum of nursing mothers at the extremes of reproductive age. PLOS ONE, 15(6), e0231882. https://doi.org/10.1371/journal.pone.0231882

Figueiredo, B., Pacheco, A., \& Costa, R. (2007). Depression during pregnancy and the postpartum period in adolescent and adult Portuguese mothers. Archives of Women's Mental Health, 10(3), 103-109. https://doi.org/10.1007/s00737-007-0178-8

Finnbogadóttir, H., \& Thies-Lagergren, L. (2017). Breastfeeding in the context of domestic violence-a cross-sectional study. Journal of Advanced Nursing, 73(12), 3209-3219. https://doi.org/10.1111/jan.13339 
Galvão, C. M. (2006). Níveis de evidência. Acta Paulista de Enfermagem, 19(2), 5-5. https://doi.org/10.1590/s0103-21002006000200001

Gershon, R. R. M., Karkashian, C. D., Vlahov, D., Kummer, L., Kasting, C., Green-McKenzie, J., Escamilla-Cejudo, J. A., Kendig, N., Swetz, A., \& Martin, L. (1999). Compliance with universal precautions in correctional health care facilities. Journal of Occupational \& Environmental Medicine, 41(3), 181-189. https://doi.org/10.1097/00043764-199903000-00007

Goyatá, S. L. T., Avelino, C. C. V., Santos, S. V. M. dos, Souza Junior, D. I. de, Gurgel, M. D. S. L., \& Terra, F. de S. (2016). Efeitos da acupuntura no tratamento da ansiedade: Revisao integrativa. Revista Brasileira de Enfermagem, 69(3), 602-609. https://doi.org/10.1590/0034-7167.2016690325i

Guyatt, G. H., Oxman, A. D., Kunz, R., Falck-Ytter, Y., Vist, G. E., Liberati, A., \& Schünemann, H. J. (2008). Going from evidence to recommendations. BMJ, 336(7652), 1049-1051. https://doi.org/10.1136/bmj.39493.646875.ae

Guyatt, G. H., Oxman, A. D., Kunz, R., Vist, G. E., Falck-Ytter, Y., \& Schünemann, H. J. (2008). What is "quality of evidence" and why is it important to clinicians? BMJ, 336(7651), 995-998. https://doi.org/10.1136/bmj.39490.551019.be

James, J. P., Taft, A., Amir, L. H., \& Agius, P. (2014). Does intimate partner violence impact on women's initiation and duration of breastfeeding?. Breastfeeding review : professional publication of the Nursing Mothers' Association of Australia, 22(2), 11-19.

Kendall-Tackett, K. A. (2007). Violence against women and the perinatal period. Trauma, Violence, \& Abuse, 8(3), 344-353. https://doi.org/10.1177/1524838007304406

Kjerulff Madsen, F., Holm-Larsen, C. E., Wu, C., Rogathi, J., Manongi, R., Mushi, D., Meyrowitsch, D. W., Gammeltoft, T., Sigalla, G. N., \& Rasch, V. (2019). Intimate partner violence and subsequent premature termination of exclusive breastfeeding: A cohort study. PLOS ONE, 14(6), e0217479. https://doi.org/10.1371/journal.pone.0217479

Krug, E. G., Mercy, J. A., Dahlberg, L. L., \& Zwi, A. B. (2002). The world report on violence and health. The Lancet, 360(9339), $1083-1088$. https://doi.org/10.1016/s0140-6736(02)11133-0

Martin-de-las-Heras, S., Velasco, C., Luna-del-Castillo, J., \& Khan, K. (2019). Breastfeeding avoidance following psychological intimate partner violence during pregnancy: A cohort study and multivariate analysis. BJOG: An International Journal of Obstetrics \& Gynaecology, 126(6), 778-783. https://doi.org/10.1111/1471-0528.15592

McFarlane, J., Greenberg, L., Weltge, A., \& Watson, M. (1995). Identification of abuse in emergency departments: Effectiveness of a two-question screening tool. Journal of Emergency Nursing, 21(5), 391-394. https://doi.org/10.1016/s0099-1767(05)80103-5

McGuire, S. (2015). World Health Organization. Comprehensive implementation plan on maternal, infant, and young child nutrition. Geneva, Switzerland, 2014. Advances in Nutrition, 6(1), 134-135. https://doi.org/10.3945/an.114.007781

Mendes, K. D. S., Silveira, R. C. de C. P., \& Galvão, C. M. (2008). Revisão integrativa: Método de pesquisa para a incorporação de evidências na saúde e na enfermagem. Texto \& Contexto - Enfermagem, 17(4), 758-764. https://doi.org/10.1590/s0104-07072008000400018

Miller-Graff, L. E., Ahmed, A. H., \& Paulson, J. L. (2018). Intimate partner violence and breastfeeding outcomes in a sample of low-income women. Journal of Human Lactation, 34(3), 494-502. https://doi.org/10.1177/0890334418776217

Moraes, L. L. de, Campos, L. D. A., Ferrari, D. V. de J., Saeki, E. K., Polettini, J., \& Morceli, G. (2019). Impacto da idade materna na acidez do colostro de nutrizes em maternidade do interior do Estado de São Paulo. Journal of Human Growth and Development, 29(2), 153-160. https://doi.org/10.7322/jhgd.v29.9414

Normann, A. K., Bakiewicz, A., Kjerulff Madsen, F., Khan, K. S., Rasch, V., \& Linde, D. S. (2020). Intimate partner violence and breastfeeding: A systematic review. BMJ Open, 10(10), e034153. https://doi.org/10.1136/bmjopen-2019-034153

Organização das Nações Unidas (ONU). Transformando Nosso Mundo: A Agenda 2030 para o Desenvolvimento Sustentável, 2015.

Organização Mundial da Saúde. Global and Regional Estimates of Violence Against Women: Prevalence and Health Effects of Intimate Partner Violence and Non-Partner Sexual Violence. Geneva: OMS, 2013.

Organização Mundial Da Saúde. Relatório mundial sobre prevenção da violência. Washington DC: OMS, 2014.

Palhoni, A. R. G., Amaral, M. A., \& Penna, C. M. M. (2014). Representations of violence against women and its relationship to their quality of life. Online Brazilian Journal of Nursing, 13(1). https://doi.org/10.5935/1676-4285.20144286

Rea, M. F. (2004a). Os benefícios da amamentação para a saúde da mulher. Jornal de Pediatria, 80(5). https://doi.org/10.1590/s0021-75572004000700005

Rea, M. F. (2004b). Os benefícios da amamentação para a saúde da mulher. Jornal de Pediatria, 80(5). https://doi.org/10.1590/s0021-75572004000700005

Santos, C. M. da C., Pimenta, C. A. de M., \& Nobre, M. R. C. (2007). The PICO strategy for the research question construction and evidence search. Revista Latino-Americana de Enfermagem, 15(3), 508-511. https://doi.org/10.1590/s0104-11692007000300023

Saúde, Brasil. M. da. (n.d.). Guia alimentar para crianças menores de 2 anos.

Silverman, J. G., Decker, M. R., Reed, E., \& Raj, A. (2006). Intimate partner violence around the time of pregnancy: Association with breastfeeding behavior. Journal of Women's Health, 15(8), 934-940. https://doi.org/10.1089/jwh.2006.15.934

Sørbø, M. F., Lukasse, M., Brantsæter, A.-L., \& Grimstad, H. (2015). Past and recent abuse is associated with early cessation of breast feeding: Results from a large prospective cohort in Norway. BMJ Open, 5(12), e009240. https://doi.org/10.1136/bmjopen-2015-009240 
Research, Society and Development, v. 11, n. 1, e18111124493, 2022

(CC BY 4.0) | ISSN 2525-3409 | DOI: http://dx.doi.org/10.33448/rsd-v11i1.24493

Souza, M. T. de, Silva, M. D. da, \& Carvalho, R. de. (2010). Integrative review: What is it? How to do it? Einstein (São Paulo), 8(1), 102-106. https://doi.org/10.1590/s1679-45082010rw1134

Tesfahun, F., Worku, W., Mazengiya, F., \& Kifle, M. (2014). Knowledge, perception and utilization of postnatal care of mothers in Gondar Zuria District, Ethiopia: A cross-sectional study. Maternal and Child Health Journal, 18(10), 2341-2351. https://doi.org/10.1007/s10995-014-1474-3

Tran, L. M., Nguyen, P. H., Naved, R. T., \& Menon, P. (2020). Intimate partner violence is associated with poorer maternal mental health and breastfeeding practices in Bangladesh. Health Policy and Planning, 35(Supplement_1), i19-i29. https://doi.org/10.1093/heapol/czaa106

Victora, C. G., Bahl, R., Barros, A. J. D., França, G. V. A., Horton, S., Krasevec, J., Murch, S., Sankar, M. J., Walker, N., \& Rollins, N. C. (2016a). Breastfeeding in the 21st century: Epidemiology, mechanisms, and lifelong effect. The Lancet, 387(10017), 475-490. https://doi.org/10.1016/s0140-6736(15)01024-7

Victora, C. G., Bahl, R., Barros, A. J. D., França, G. V. A., Horton, S., Krasevec, J., Murch, S., Sankar, M. J., Walker, N., \& Rollins, N. C. (2016b). Breastfeeding in the 21 st century: Epidemiology, mechanisms, and lifelong effect. The Lancet, 387(10017), 475-490. https://doi.org/10.1016/s0140-6736(15)01024-7 